\title{
Research on Agent-based Human-Information System Trusted Interaction in Distributed Cooperative Work Environment
}

\author{
Lin Zhang ${ }^{1,2, *}$, Shu Ping $\mathrm{Yi}^{1}$ and Run Zhao ${ }^{1}$ \\ ${ }^{1}$ School of Mechanical Engineering, Chongqing University, Chongqing 400030, China \\ ${ }^{2}$ College of Computer and Information Science, Southwest University, Chongqing 400715, China
}

\begin{abstract}
In order to solve the problem of untrustworthy interactive process in distributed cooperative work environment, this paper, firstly, proposes a notion of "human-information system trusted interaction" and provides the basic research framework. Secondly, this paper illustrates the meaning of this notion and studies the characteristics of the trustworthiness about the human-information system trusted interaction. Finally, we analyses the key factors influencing the human-information system trusted interaction and puts forward a three-layer trustworthy interactive model based on agent. A trustworthy interaction management layer is inserted between users and information system. The management layer includes 5 agents. The Interaction agent and negotiation agent track and collect data of interactive behavior; monitor agent maintains global clock to guaranty temporal consistency; assessment and analysis agents used for determining abnormal interactive behaviors; and feedback agent is used for giving user prompt and early warning. To use this model, we constructed a trustworthy interaction chain of human-information system. Due to the each link of the chain is monitored and managed by the management layer, the trustworthiness of interaction process is guaranteed.
\end{abstract}

Keywords: Cooperative Interaction, Distributed cooperative, Agent, Trustworthy interaction.

\section{INTRODUCTION}

"Trustworthiness" means the behavior of an entity always in line with people's expectations when it achieving objectives and it focus on the consistency between objectives and realization, and testability and controllability of the behavior. With the rapid development of information technology, software system has been applied in almost every field of national economy and defense, which makes it play an increasingly important role in the information society. However, as the software scale and complexity are growing, information system has become more and more fragile. The diversity of software faults and failures will cause huge losses to the state, enterprises and users. This phenomenon is called the trustworthiness problem of the software [1]. To solve this problem in critical field, at the end of 2007, the National Natural Science Foundation of China launched a major research plan of trustworthy software. How to efficiently develop and operate trustworthy software systems has become a major scientific problem in both information and management fields.

In the use of information systems, the results of trustworthiness are not only affected by the trustworthiness of the software and its running environment, but also by the trustworthiness of interactive process between human and information system [1]. Nowadays, more and more people work in distributed environments which characterized by spatial distribution, time synchronous or asynchronous, heterogene

*Address correspondence to this author at the School of Computer \& Information Science, Southwest University, Chongqing, 400715, P. R. China; Tel: +86-023-68252352(office), +86-13647690009 (Mobile); Fax: +86-023-68252051; E-mail: fly_fox2005@163.com ous environment, and supported by information technology. In this environment, human and information system can share data, information and knowledge, collaborate with other people and information system to complete some specific tasks [2, 3]. At present, great deal of researches have been done in distributed environment, process and methods. These studies promoted the development of distributed cooperative work, but the effects of collaborative work have not been very satisfactory. There still exist some issues such as interactive delay, restructuring of interactive process, unreliable information channel, and different information format caused by geographical distribution, heterogeneous platforms, concurrency, and collaborativity, which would lead to the collaboration failure and untrustworthy results. For example, in the financial field, there exists a remarkable phenomenon that engenders huge losses for banks owing to human interaction errors; and Olympic ticket sales system has broken down due to the out-of-order interaction of the large number of people. Therefore, the research of "humaninformation system trusted interaction" is becoming a new scientific issue to be resolved, it is developing as a new study direction of trusted computing and trusted software, and it is also starting a new research field for the availability of human-computer.

\section{CURRENT SITUATION ANALYSES}

At present, most the correlation researches of "humaninformation system trusted interaction" are focused on three aspects that are as follows:

\section{(1) The Human-Computer Interaction}

Some researchers synthesize the human's action, Cognitive Model, Operation Model, Distributed Cognitive Model, 
Thinking Model [4-7], Information Processing and Information Interactive Model [8, 9] and User Trust Model [10] to put forward a principle that we should consider the behavior of human's Cognitive and receive information when designing the IS interface, and attach importance to the users' predictable behavior of interaction [11], and to improve the reliability and ability of Human's information Processing during the human-computer interaction. Some other researchers analyze the types of operation errors in the human-computer system to advance many reasonable proposals and measures about interaction interface design that can reduce errors and improve the efficiency of cognitive [12-15]. Many scholars do more research on the matching model between human, task and information to provide the factors and mechanism that influence H-IS interaction efficiency [16, 17]. Currently, a number of researchers, according to the task characteristics, work environment and interaction tools, try to improve interaction channels [18], bring forward analysis model of group task collaboration [19, 20] and ameliorate interaction strategy facing the resources and environment [21] to coordinate, control and use shared resources, and then to advance the interactive characteristic and availability during the H-IS interaction.

\section{(2) The Information System Trustworthiness}

Some researchers consider the software trustworthiness [22-24], from the aspect of software development and software component [25], and analyze the software aging and information failure, and then use some advanced new models and methods which can improve information system design and heighten the reliability of IS running. Aiming at the safe problem of IS use, some other researchers probed into the security of IS running [26, 27], though the side of firewall, agent technology and CA certification. From the angle of trust, many scholars use mathematics model of trusted computing and trusted computing platform alliance technology to study the trust relationship of various roles between IS and network, and then established trust level model to ensure interaction of security between users and IS [28, 29].

\section{(3) Trustworthiness of Distributed Cooperative Work}

Mr. Long put forward a collaboration model consisting client view, security control, whole character and network. It ensures the operational safety of users [30]. From the point of internal data exchange of collaborative system, Mr. Fang studied poor integration of systems and unreliable Cooperative [31]. Mr. Zhang put forward trusted computing-based access control architecture for CSCW (Computer Supported Cooperative Work) and roles-based delegation policy between collaboration workstations [32]. $\mathrm{Mr}$. $\mathrm{Hu}$ proposed a collaboration interactive model based on intelligent agent [33]. Mr. Zhou put forward a model of multi-Agent group (MAG) for monitoring and controlling hazard installations [34]. Sergio Ilarri proposed a loose and fault-tolerant monitoring approach based on mobile agents for tracking of the involved computers, carrying the monitoring tasks [35].

Through a comprehensive analysis of extensive literature, distributed cooperative work environment (DCWE is for short) and interactive process of H-IS, we found that the following problems in interactive process of H-IS in DCWE have still not been solved.
(1). The asymmetry of human ambiguity and accuracy of information system can lead to untrustworthy interaction between human and information systems;

(2). The conflict between the randomness of human behavior and the temporal logic of information system can induce untrustworthy collaboration between human and information systems;

(3). Interferences, such as errors, environmental impacts, external attacks, cause the data and information to become non-credible in interactive process;

(4). Heterogeneous and stochastic of environment can cause untrustworthy interaction between information systems.

In order to solve the above problems, and to improve trustworthiness of interactive process, the trustworthy interaction chain (TIC) model of H-IS based on agent is firstly proposed by combining the characteristics of DCWE and interactive process of H-IS.

\section{THE MEANING AND CHARACTERISTICS OF THE H-IS TRUSTED INTERACTION}

\subsection{The Meaning of the H-IS Trusted Interaction}

The meaning of H-IS trusted Interaction is that: under the distributed working environment, using the computer for support platform and with the IS as the object, in order to achieve the aim, which is the trusted interaction process that includes some high trustworthiness characteristics, such as reliability, cooperativity, testability, timeliness, security, availability and so on, and during this process, human and human, human and IS need to continuously process and exchange information effectively and collaboratively. And then the trusted interaction process can provide benefit by advancing the degree of trustworthiness of IS running result.

Because of the impact of the characteristic of task, environment, and the users' needs, the purpose of H-IS interaction is not only completing a certain task effectively, but also considering doing while various abnormities accurately to execute the whole process with high trustworthiness even if the IS, which we use during the interaction process, is quite complicated. Right now, many large and complex ISs are used in several application field, such as the Financial Industry, Business Industry and so on. Because those work places are distributed, this interaction needs to be administered collaboratively by a number of people instead of a single worker. Thus, the intricate and cooperative interaction results in the difficulties of information transfer communication and understanding. And all of these bring about the challenge for $\mathrm{H}$-IS trusted interaction.

\subsection{The Characteristics of the H-IS Trusted Interaction}

$\mathrm{H}$-IS trusted interaction is a complex, dynamic and collaborative process with trusted running of IS as the aim. Based on the analysis of representative cases and the research of interrelated theories, the author classifies this process into three parts: Information Processing Model, Single Human-Information System collaborative Interaction Model, and Multi-Human-Information System collaborative Interaction Model under the distributed working environment (The 


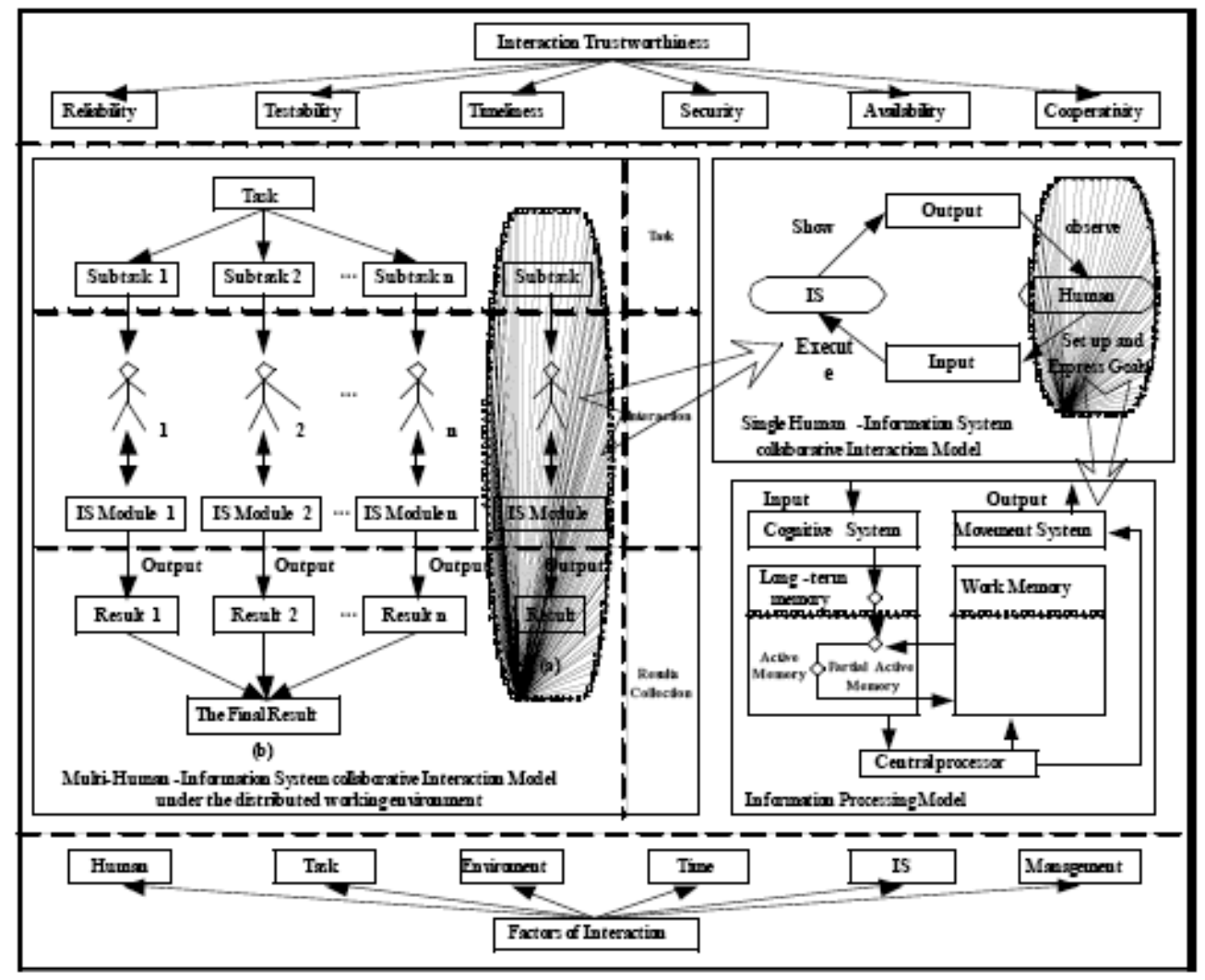

Fig. (1). Human-Information System Collaborative Interaction Model under the Distributed Working Environment.

frame is showed in the Fig. 1). The characteristics of this process are as follows:

(1). The human is the main part and the crucial factor of $\mathrm{H}-\mathrm{IS}$ trusted interaction. And whatever the IS has so the high and intricate intelligence, the human function cannot be ignored during the interaction. In the context of information technology widely used, the research of H-IS trusted interaction is not different from the former study of human-machine in which the human is the main part and the machine is the tool, and is to analyze the cooperative interaction between the knowledge workers and IS with certain intelligence.

(2). There exists interactive characteristics during the H-IS trusted interaction process. Under the distributed working environment, human and human, human and IS exchange lots of information, and both sides must respond with each other. It emphasizes the interaction, but not control or surveillance of one another: the inputs of information by each side need the others to deal with the information automatically and give the right output. The different results can also bring along the new interaction between the two sides. And lots of information will be showed to workers during each interaction and the human must make a right decision in time.

(3). H-IS trusted interaction has the cooperative characteristics. The interaction task cannot be completed independently by singe worker or IS. It needs human and human, human and IS, IS module and IS module, department and department to work cooperatively. Dur- ing this process, there also exits the collaboration between the cognitive organ and operation organ of human, and exits the group cognitive cooperation in the sharing work space.

(4). H-IS trusted interaction has the dynamic characteristics. The interaction process is not immobile, but is evolving ceaselessly as time goes on. The dynamic changes are primarily arisen by the Time-Variation of all kinds of factors affecting the trusted interaction. Because of the interactive characteristics the trusted interaction can take on the alternation between continuity and discreteness.

(5). H-IS trusted interaction has the distributed-type characteristics. At present, in same critical fields, the large and complex IS is operated cooperatively by many people. Thus, under this situation, the people work places should be distributed during the H-IS interaction. And the Multi-Human-Information System collaborative Interaction can be considered as a synthesis that is composed of many Single Human-Information System collaborative Interaction models.

(6). The trustworthiness is the most important characteristics. There are six attributes as follows:

(1) Reliability: During the interaction process, with the high reliable operation behavior, task execution behavior and business administration flow, human are engaged with information processing and disposition, and possesses the ability that can ensure that there is no invalid interaction under the stated environment. 


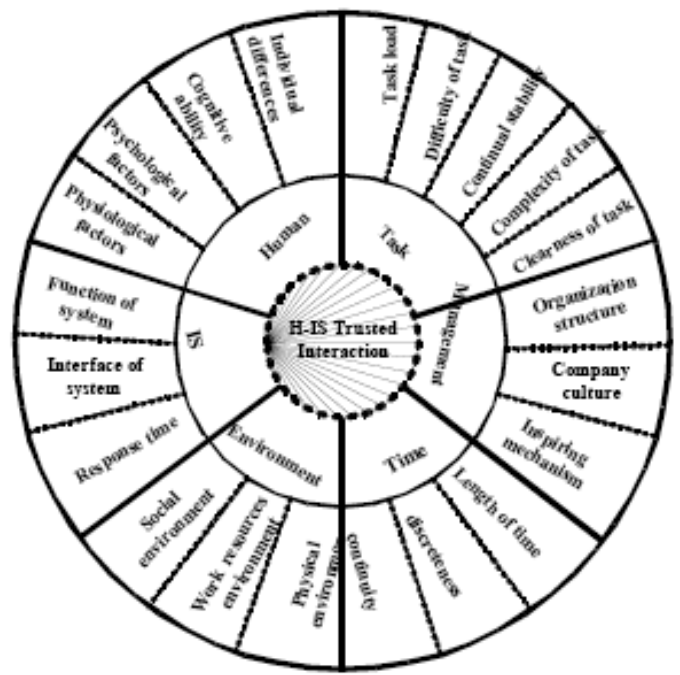

Fig. (2). The Types of Factors Affecting the H-IS Trusted Interaction.

(2) Testability: During the normal running of IS, Human has an ability that can avoid, prevent, detect and diagnose interactive obstacles and adjust the interaction behavior.

(3) Timeliness: Human can process information and maintain information value-added in manner of fast response.
(5) Availability: Effectiveness, i.e. the degree of correctness and integrality that human complete the special task and achieve given goals; Efficiency, i.e. the ratio of the degree of correctness and integrality to the recourses used in the interaction; Satisfaction, i.e. the degree of subjective satisfaction and acceptance that is gotten in the interaction.

(6) Cooperativity: During the interaction process, human and human, human and IS can cooperatively execute many intricate task all together.

\section{FACTORS AFFECTING THE H-IS TRUSTED INTERACTION}

At the present time, there are not really cooperativity and trustworthiness in the H-IS interaction process. Though the factors of Affecting the H-IS Trusted Interaction are too many, we should understand these factors more clearly so that we can be better to manage and control the interaction and then advance the degree of trustworthiness about the $\mathrm{H}$ IS interaction. Based on the earlier study, the analyses of representative cases and actuality investigation, the author divide the factors into six types (These factors are showed in the Fig. (2) clearly): human factor, task factor, management factor, environment factor, IS factor and time factor.

We can understand the meaning and characteristics of the $\mathrm{H}$-IS trusted interaction basically by the analysis of six main

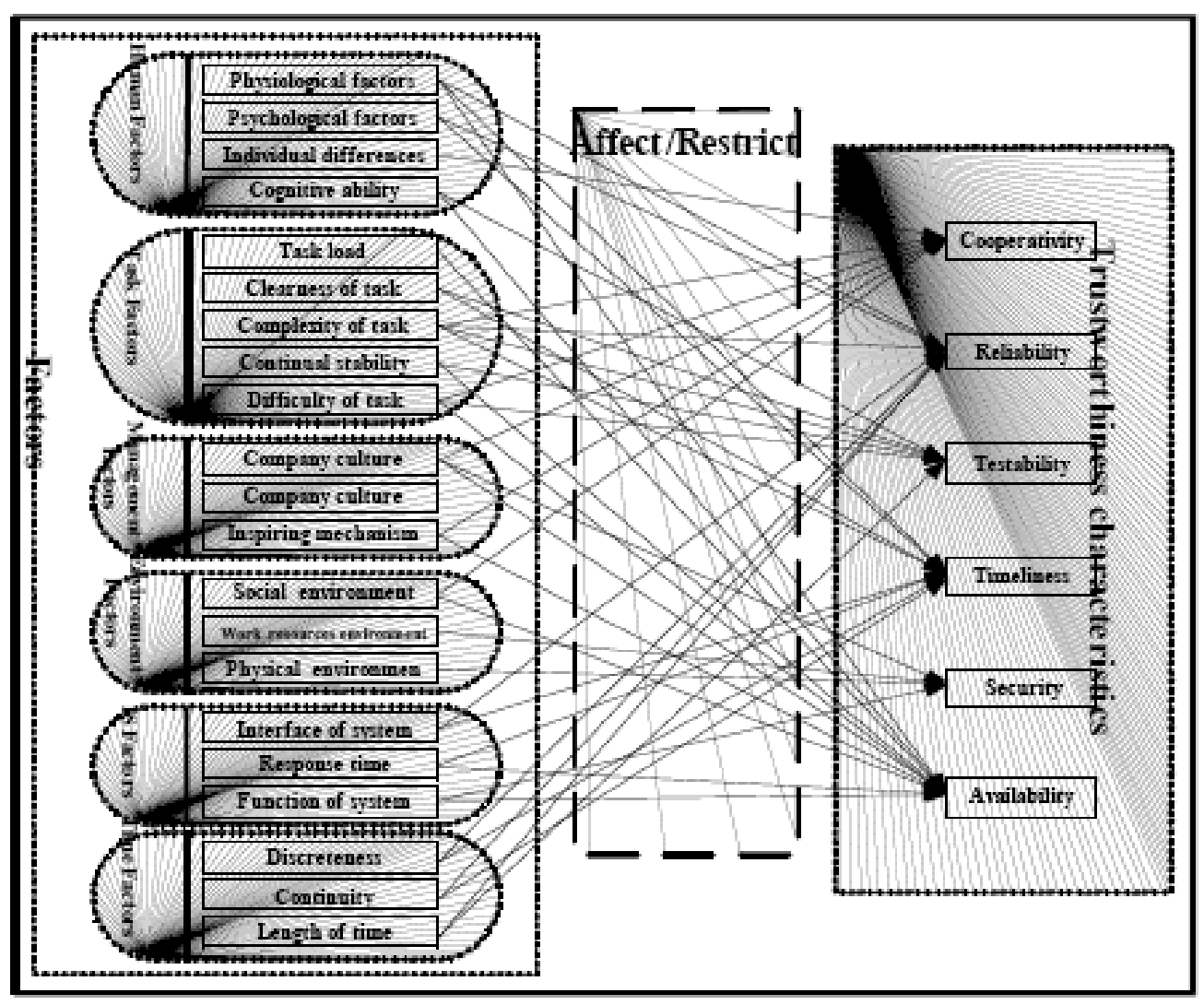

Fig. (3). The Relationship between Factors and Characteristics.

(4) Security: Human can ensure the whole interaction process is executed under the secure environment, and can interact with IS in security. factors affecting the trusted interaction. In order to visualize the relationship between factors and characteristics, the author uses the Fig. (3) to illuminate it (Fig. 3). 


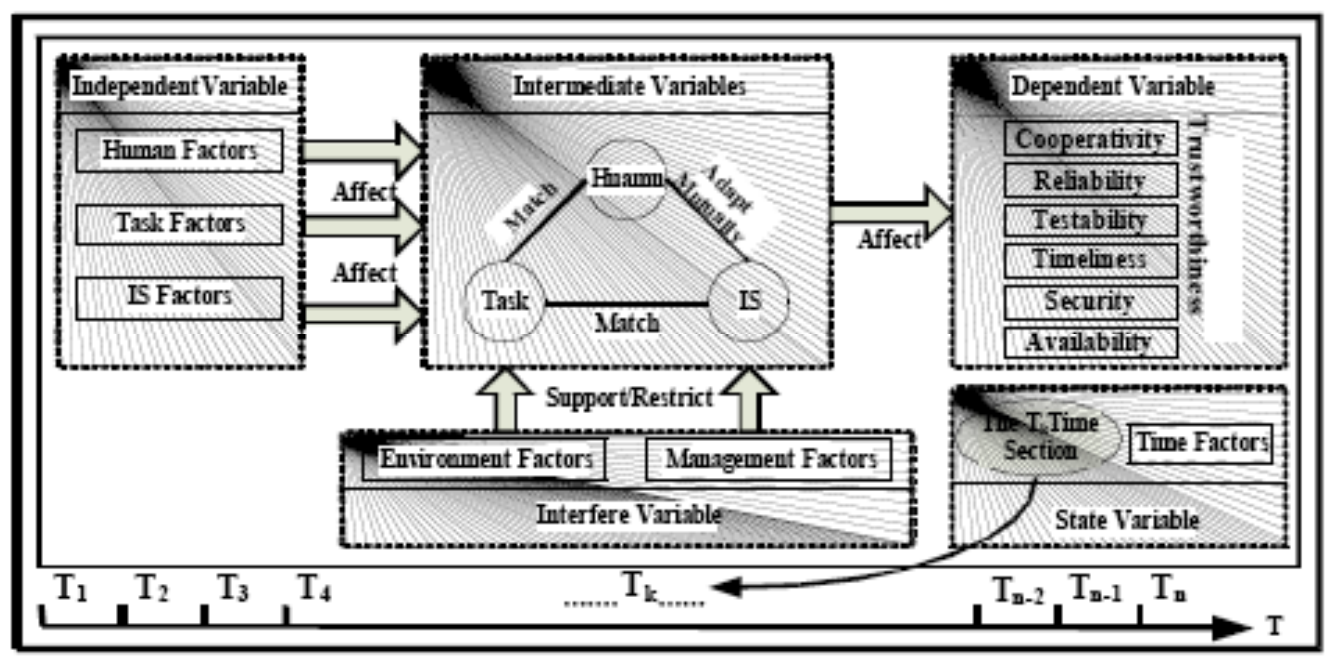

Fig. (4). The Model of the H-IS Trusted Interaction Based on the Dynamic Matching.

\section{THE DYNAMIC MATCHING MODEL OF THE H-IS TRUSTED INTERACTION}

The factors of the H-IS trusted interaction cannot restrict the trusted interaction respectively, and there exists the restriction relationships, which show the dynamic variability with the time, among the factors. For example, the analysis of task can be affected by management factors, the human reliability depends on support of environment, and the time changing can bring about time-varying of intertwining relationship of the other factors. There are certain logistic matching relationships affecting the process of interaction among the factors all together. Therefore, in order to study the interaction mechanism of the factors, the author constructs the relative model to give a preliminary illumination about it (Fig. 4).

Under the different time sections, Task, human and IS are the basis factors of the H-IS trusted interaction all the while. The matching relationship of them is the decisive to the trusted interaction, and the environment and management play a supporting role during the interaction. Thereby, the author set up a dynamic matching model, in which the interaction process is considered as that it is made up of $n$ discrete time sections, and which is based on time as the abscissa.

In the Fig. (4), we can see that: (1) Human factors, task factors and IS factors are the independent variable; (2) Environment Factors and management factors are the interfere variable; (3) The matching of "Human-Task-IS" is the intermediate variables; (4) The trustworthiness characteristics is the dependent variable; (5) Tine is the state variable.

\section{THREE-LAYER TRUSTWORTHY INTERACTIVE MODELBASED ON AGENT}

In order to avoid abnormal behavior caused by errors or malice, and also to ensure the trustworthiness of interaction process, we build a three-layer model of trustworthy interaction. The three-layer concept model of trustworthy interaction includes information system layer, trustworthy interaction management layer and user layer (refer with: Fig. 5). The management layer is the core of the model, which is responsible for the management of interaction of H-IS and

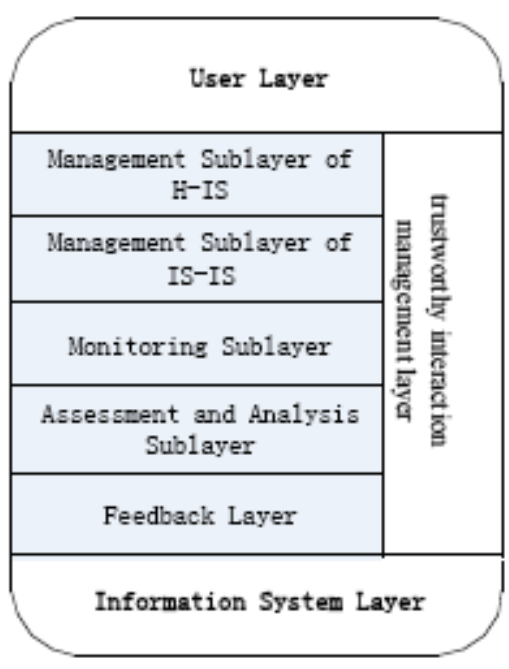

Fig. (5). Three-layer concept model of trustworthy interaction.

among information systems, tracking and collection of interaction data, assessment and analysis of the collected data, and feedback the result of the evaluation to the user.

Since agent technology has been proved to be very suitable for the problem of the interaction modeling, intelligent agent technology will be used to build intelligent interaction agents which have different intentions, cognitive ability, knowledge base, behavior strategy, and decision mode. And then, we established trustworthy interactive model of H-IS based on these agents (refer with: Fig. 6).

In this model, interaction agent and consultation agent are responsible for data collection of interaction behavior; monitor agent maintain global clock to guaranty temporal consistency; assessment and analysis agent is used for determining whether abnormal interactive behaviors; and feedback agent used for giving is user prompt and early warning.

\section{TRUSTWORHY INTERACION CHAIN BASED ON THE THREE-LAYER MODEL}

Within distributed collaborative work environment, trustworthiness of interaction process is not simple integration of trustworthy interaction acts and nodes. And it needs 


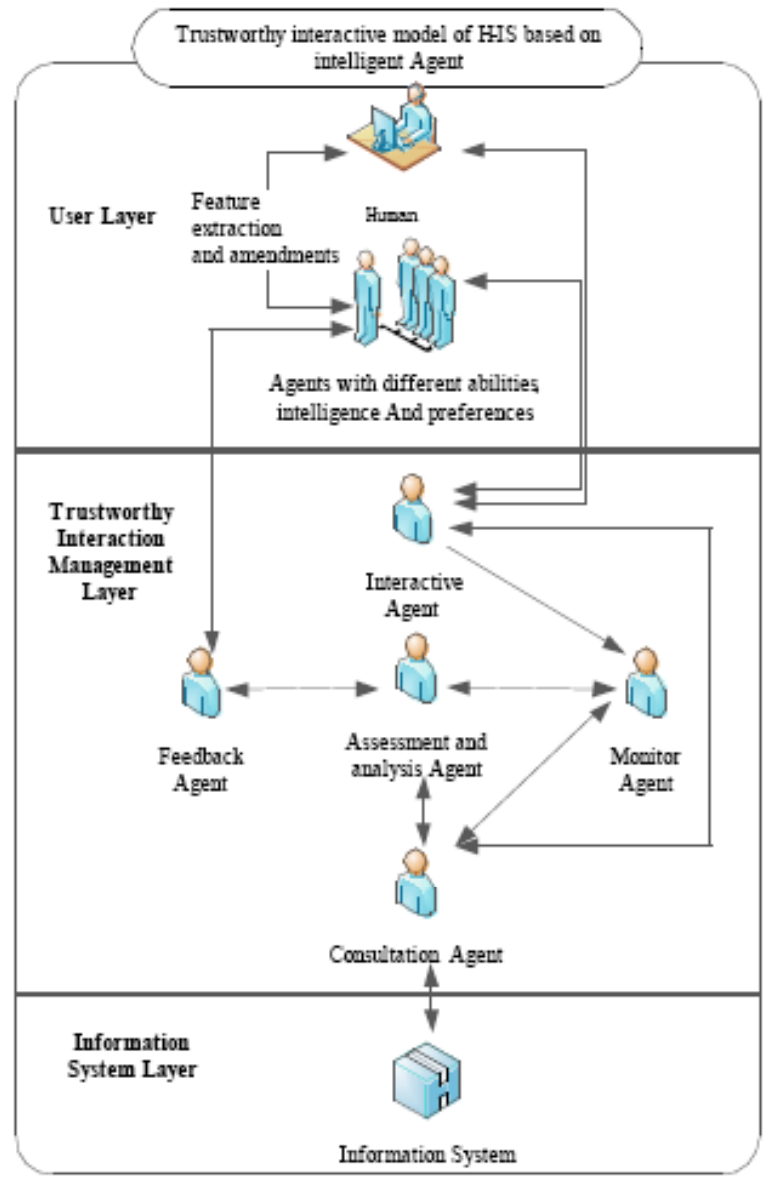

Fig. (6). Trustworthy interactive model of H-IS based on intelligent agent.

interwork of these acts and nodes to compose a specific interaction sequence with specific temporal and spatial logic. We called the sequence interaction chain.

Compared with the interaction acts and nodes, interaction chain has more prominent effect on trustworthiness of interaction results. Based on the above understanding and combined with the three layer mode and interaction chain, we build a trustworthy interaction chain. The TIC has a star topology (refer with: Fig. 7). In implement, TIC usually has variety of logical structure such as linear, tree, ring and mesh (refer with: Fig. 8). The letter M, in the Fig. (4), stands for instance of trustworthy interaction model based on intelligent agent.

Each interaction act and node of TIC is monitored by trusted agents. This can ensure trustworthiness of entire interactive process under the distributed cooperative work environment.

\section{CONCLUSIONS}

Trustworthiness of interaction process is not simple integration of trustworthy interaction acts and nodes. In order to ensure the trustworthiness of interaction process, we build a three-layer model of trustworthy interaction, and construct TIC of H-IS based on intelligent agent technology.

At present, although we have established the TIC model based on intelligent agent, but in distributed cooperative

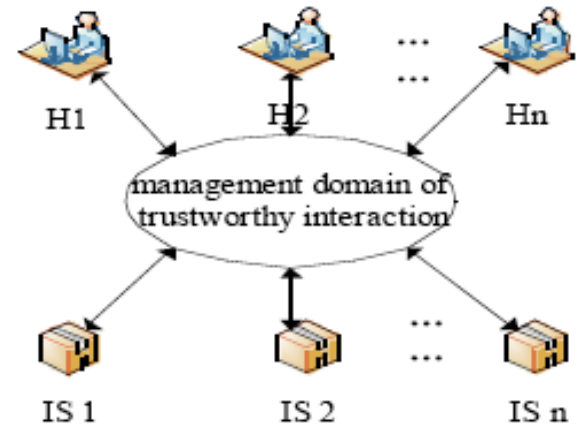

Fig. (7). Star topology of TIC.

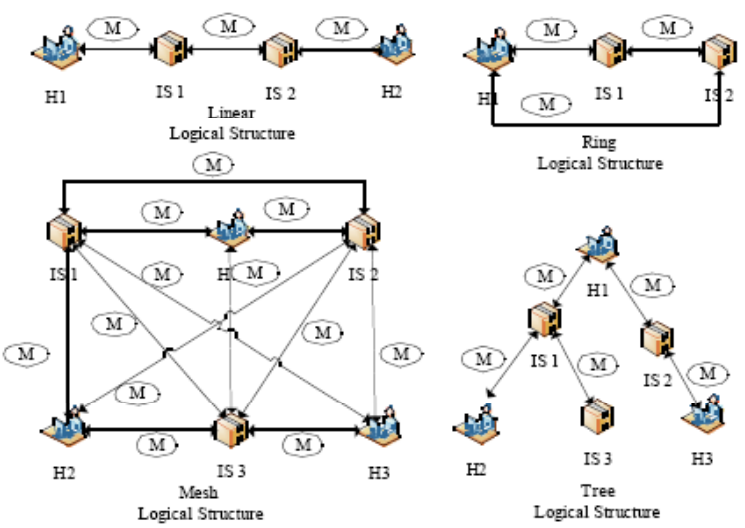

Fig. (8). Logical structure of TIC.

work environment, the application of TIC still faces the following problems:

(1). The relationship and mechanism of the factors influencing the H-IS trusted interaction is clear;

(2). How to mine the mechanism and validate the matching model from at the experiment angle;

(3). How to quickly adapt to dynamic changes of interactive process in the TIC;

(4). How to evaluate and optimize trustworthiness of interaction process.

Further study will be continued to solve these problems in several typical distributed cooperative work environments, such as network manufacturing.

\section{ACKNOWLEDGMENTS}

The author thanks the editors and the anonymous reviewers for their helpful comments and suggestions. This research is funded by Chongqing Natural Science Foundation (The project No. is CSTC, 2009BB2308).

\section{CONFLICT OF INTEREST}

None Declared.

\section{REFERENCES}

[1] L. Ke, S. Zhiguang, W. Ji, H. Jifeng, Z. Zhaotian, and Qn Yuwen, "Overview on Major Research Plan of Trustworthy Software". Bulletin of National Natural Science Foundation of China, vol.22, no. 3, pp. 145-151, 2008

[2] J. Dan-dong, Z. Shen-sheng, and C. Lu, “Research on Distributed Virtual Cooperative Working Environment and the Implementa- 
tion', Journal of Computer Engineering and Applications, vol. 9, pp. 13-16, 2002.

[3] M. Samsonova, A. Pisarev, K. Kozlov, E Poustelnikova, and A. Tkachenko, "An information management system for collaboration within distributed working environment", Journal of Proceedings of Data Integration in the Life Sciences, DILS, LNBI 4075, pp. 204-215, 2006.

[4] S. Linyan, L. Zhixiao, and J. Tianshi, "Cognitive Synthetic Model and Its Application in HCI. Journal of Xi'an Jiaotong University, vol. 31, no. (1), pp. 74-81, 1997.

[5] C.D. Weikenshi and J.G. Helanci, "Engineering Psychology and Human operations", East China Normal University Press.2004.

[6] M. John and L Carrol, "Human-computer interaction: psychology as a science of design", International Journal of Human-Computer Studies, vol. 46, pp. 501-522, 1997.

[7] W. Chang-Qing, D. Chang-Zhi, M.A. Cui-Xia, H. Qing-Yi and D. Guo-Zhong, "An Extended Resources Model Based on Distributed Cognition Theory", Journal of Software, vol.16, pp. 1717-1725, (2005).

[8] Sun Linyan. Human Factors [M]. China Science and Technology Publishing House. 2005

[9] E.G. Toms, "Information Interaction: Providing a Framework for Information Architecture", Journal of the American Society for Information Science and Technology, vol. 53, no.10, pp. 855-862, (2002).

[10] L. Larry, “Constantine, IDSA.Trusted Interaction: User Control and System Responsibilities in Interaction Design for Information Systems', CAiSE 2006, LNCS 4001, pp. 20-30, 2006.

[11] H. Alison, and E. Tony, "Human elements in information system design for knowledge workers ", International Journal of Information Management, vol. 20, pp. 297-309, (2000).

[12] M. Kitajima, P.G. Polson, "A comprehension-based model of correct performance and errors in skilled, display-based, humancomputer interaction", International Journal of Human-Computer Studies, vol. 43, no.1, pp. 65-99, 1995.

[13] P. Beynon-Davies, "Human error and information systems failure:the case of the London ambulance service computer-aided despatch system project', Interacting with Computers, vol. 11, no. 6, pp. 699-720, 1999.

[14] L. Baofeng, S. Bihui and X. Hongjun, "The Design Measures of Human-Machine Interface Based on Human Factors ", Chinese Journal of Ergonomics, vol. 12, no. 1, 2006.

[15] S. Siu-Tsen, W. Martin and P. Stephen, "Towards culture-centred design", Interacting with Computers, vol. 18, no. 4, pp. 820-852, 2006.

[16] Y.Wencai, YI Shuping and T. Xiaoying, "Exploring humaninformation system interaction efficiency within the enterprise computerization environment', Proceedings of the 12th International Conference on Industrial Engineering and Engineering Management, Chongqing, pp. 577-582, 2005.

[17] YI Shuping and Y. Wencai, "Influencing Factors and Action Mechanism of Human-IS Interactive Efficiency within Enterprise Computerization Environment', Proceedings of the 7th AsiaPacific Conference on Computer Human Interaction, Taipei, Taiwan, China.10 pp.11-14, 2006.
[18] D. Shihai, "'Progress and Challenge of Human-Computer Interaction", Journal of Computer-Aided Design \& Computer Graphics, vol. 16 , no. 1, 2004.

[19] GC. van der Veer, C. Chisalita and M. van Welie, "Introduction to Groupware Task Analysis (GTA). Jul. 2002 Proceedings of the First International Workshop on Task Models and Diagrams for User Interface Design, pp. 32-39, 2002.

[20] G. Mori, F. PaternoÁ and C. Santoro, "Support for Developing and Analyzing Task Models for Interactive System Design", IEEE Transactions on Software Engineering, vol. 28, no. 8, pp. 797-813, 2002.

[21] Z. Mingjun, A. Xiang, D. Changzhi, T. Feng, and D. Zhongguo, "Resource Model for Collaborative Interaction Tasks", Journal of Computer-Aided Design \& Computer Graphics, vol. 19, no. 10, pp. 1321-1327, 2007.

[22] W. Yuan, J. XU, and FZ Lin, "A Trust Measurement and Evolution Model for Internetware", Journal of Software, vol. 11, no. 4, pp. 682-690, 2006.

[23] G. Rothermel, "Using source-code analysis to help end-user programmers create dependable software", Source-code analysis And Use.(2004).

[24] O. Berman and M. Cutler, "Resource allocation during tests for optimally reliable software", Computers \& Operations Research, vol. 31, no. 11, pp.1847-1865, 2004.

[25] L. Jiang and G. Xu, "Modeling and analysis of software aging and software failure", Journal of Systems and Software, vol. 80, no. 4, pp. 590-595, 2007.

[26] Trcek D, "Security Models:Refocusing on the Human Factor", Computer, vol. 39, no. 11, pp.103-104, 2006.

[27] EI-Alfy, E-S M.A., "Heuristic Approach for Firewall Policy Optimization", Advanced Communication Technology, vol. 3, pp. 1782-1787, 2007.

[28] Z.1, Yan and P. Cofta, Nokia Res.Center, Helsinki,Finland. “A mechanism for trust sustainability among trusted computing platforms", International Journal of Human-Computer Studies, vol. 62, pp. 381-422, 2005.

[29] S Ahmad-Reza, "Challenges for trusted computing. Lecture Notes in Computer Science.8th International Workshop on Cryptographic Hardware and Embedded Systems', pp. 414, 2006.

[30] L. Hongneng, W. Weimin, G. Peng, etc. "Collaboration Model for Product Development and Its Application", Journal of Computer Engineering and Applications, vol. 43 no. 20, pp. 235-239, 2007.

[31] F. Ke, and Y. Ming., Journal of Computer Simulation, vol. 21, no. (8), 194-197, 2004.

[32] Z. Zhiyong, Y. Lin, M. Jianfeng, P. Jiexin, "CSCW System Access Control Based on Trusted Computing", Journal of Huazhong University of Science and Technology (Nature Science Edition), vol. 36, no. 1, pp. 59-62, 2008.

[33] H. Yaoguang, W. Tianmiao, W. Jiashun, M. Xiaoning, and Y. Weiming, Journal of Systems Engineering and Electronics, vol. 24, no. 1, pp. 17-20, 2002.

[34] Z. Jianfeng, C. Guohua1, W. Musheng, and C. Qingguang, "MultiAgent-Based Distributed Monitoring and Controlling System for Hazard Installations", Journal of South China University of Technology (Natural Science Edition), vol. 35, no. 9, pp. 107-112, 2007.

[35] S. Ilarri, E. Mena and A. Illarramendi, Information Sciences, vol. 178, no. 9, pp. 2105-2127, 2008.

Received: September 07, 2010

Revised: November 25, 2010

Accepted: March 03, 2011

(C) Zhang et al.; Licensee Bentham Open.

This is an open access article licensed under the terms of the Creative Commons Attribution Non-Commercial License (http://creativecommons.org/licenses/by-nc/3.0/) which permits unrestricted, non-commercial use, distribution and reproduction in any medium, provided the work is properly cited. 